\title{
Avaliação inflamatória e imunohistoquímica de materiais reparadores biocerâmicos após pulpotomia: estudo em ratos wistar
}

Inflammatory and immunohistochemical evaluation of bioceramic repair materials after pulpotomy: study in wistar rats

Evaluación inflamatoria e inmunohistoquímica de materiales de reparación biocerámica después de pulpotomía: un estudio en ratas wistar

Diego Valentim

ORCID: https://orcid.org/0000-0002-5699-6578 Universidade Estadual Paulista, Brasil E-mail: dvalentim_5@hotmail.com Carlos Roberto Emerenciano Bueno ORCID: https://orcid.org/0000-0002-1897-2823 Universidade Estadual do Norte do Paraná, Brasil E-mail: dentistabueno@gmail.com Ana Maria Veiga Vasques ORCID: https://orcid.org/0000-0002-1211-2363 Universidade Estadual Paulista, Brasil E-mail: anavvasques@hotmail.com

Francine Benetti

ORCID: https://orcid.org/0000-0002-5459-353X

Universidade Federal de Minas Gerais, Brasil

E-mail: Francine Benetti@hotmail.com

Marina Tolomei Sandoval Cury

ORCID: https://orcid.org/0000-0003-0733-7936 Universidade Estadual Paulista, Brasil

E-mail: ma.tolomei@gmail.com

Ana Claudia Rodrigues da Silva ORCID: https://orcid.org/0000-0002-7570-6126 Universidade Estadual Paulista, Brasil E-mail: claudia.silva@unesp.br

Rogério Castilho Jacinto

ORCID: https://orcid.org/0000-0002-2362-8920 Universidade Estadual Paulista, Brasil E-mail: rogerio.castilho@unesp.br

Gustavo Sivieri-Araújo

ORCID: https://orcid.org/0000-0002-8402-7408 Universidade Estadual Paulista, Brasil E-mail: gustavo.sivieri@unesp.br Edilson Ervolino

ORCID: https://orcid.org/0000-0003-4859-0583 Universidade Estadual Paulista, Brasil E-mail: e.ervolino@unesp.br

Luciano Tavares Angelo Cintra ORCID: https://orcid.org/0000-0003-2348-7846 Universidade Estadual Paulista, Brasil E-mail: luciano.cintra@unesp.br Eloi Dezan Junior

ORCID: https://orcid.org/0000-0002-5699-6578 Universidade Estadual Paulista, Brasil E-mail: eloi.dezan@unesp.br

\begin{abstract}
Resumo
A pulpotomia é uma opção de tratamento conservador que têm como função preservar a vitalidade pulpar na porção radicular, com o uso de materiais biocompatíveis sobre este tecido remanescente. Este estudo teve como objetivo avaliar a resposta biológica do remanescente pulpar frente aos cimentos biocerâmicos reparadores Biodentine ${ }^{\circledR}$ e MTA Branco Angelus $^{\circledR}$ comparados ao hidróxido de cálcio após pulpotomia. Foram utilizados vinte e quatro ratos machos que tiveram as polpas coronárias do primeiro e segundo molar expostas e removidas com uma cureta afiada. $\mathrm{O}$ tecido pulpar remanescente recebeu um dos materiais experimentais: Biodentine ${ }^{\circledR}$, MTA Angelus ${ }^{\circledR}$ ou $\mathrm{Ca}(\mathrm{OH})_{2}+$ água destilada e
\end{abstract}


selados com ionômero de vidro. Um grupo foi selado diretamente com ionômero de vidro, como grupo controle negativo. Após 7 e 15 dias os animais foram eutanasiados e as peças submetidas ao processamento histológico para avaliação do processo inflamatório pela colocaração em HE e imunoistoquímico (Fibronectina e Tenascina), através da atribuição de scores de 1 a 4 . Formação de ponte de tecido duro foi observada em coloração HE, avaliando presença, continuidade e morfologia. Os dados foram submetidos ao teste de Kruskal Wallis e Dunn (p<0,05). A análise estatística mostrou que aos 7 dias o MTA e o $\mathrm{Ca}(\mathrm{OH})_{2}$ tiveram maior continuidade da ponte de tecido duro do que o ionômero de vidro $(\mathrm{p}<0,05)$. O Biodentine ${ }^{\circledR}$ apresentou melhores aspectos morfológicos em relação ao ionômero de vidro $(\mathrm{p}<0,05)$. Aos 15 dias o MTA e o Biodentine apresentaram ponte de tecido duro completa $(\mathrm{p}<0,05)$. Para a imunomarcação, o Biodentine $^{\circledR}$ obteve maior marcação que o ionômero de vidro para Fibronectina e Tenascina. O Biodentine ${ }^{\circledR}$, o MTA Angelus Branco e o hidróxido de cálcio apresentaram capacidade de induzir mineralização perante a metodologia aplicada. No entanto, o Biodentine ${ }^{\circledR}$ mostrou melhor resposta tecidual que o ionômero de vidro e o hidróxido de cálcio. Palavras-chave: Pulpotomia; Inflamação; Teste de materiais; Endodontia.

\begin{abstract}
Pulpotomy is an alternative of conservative treatment which aims to preserve pulp vitality within root portion, with the aid of biocompatible materials on this remaining living tissue. This study aimed to evaluate the biological response of the pulp remnant to Biodentine ${ }^{\circledR}$ and white Angelus MTA ${ }^{\circledR}$ bioceramic repair cements compared to calcium hydroxide after pulpotomy. Twenty-four male rats had the coronary pulps of the first and second molars exposed and removed with a sharp curette. The remaining pulp tissue received one of the experimental materials: Biodentine ${ }^{\circledR}$, white Angelus $\mathrm{MTA}^{\circledR}$ or $\mathrm{Ca}(\mathrm{OH}) 2+$ distilled water and sealed with glass ionomer. One group was sealed directly with glass ionomer as a negative control group. After 7 and 15 days, the animals were euthanized and the pieces submitted to histological processing to evaluate the inflammatory process with HE staining and immunohistochemistry (Fibronectin and Tenascin), through the attribution of scores from 1 to 4 . Formation of hard tissue bridging was observed in HE staining, evaluating presence, continuity and morphology. Data were submitted to the Kruskal Wallis and Dunn test $(\mathrm{p}<0.05)$. Statistical analysis showed that at 7 days MTA and $\mathrm{Ca}(\mathrm{OH}) 2$ had greater hard tissue bridge continuity than glass ionomer $(\mathrm{p}<0.05)$. Biodentine ${ }^{\circledR}$ showed better morphological aspects compared to glass ionomer $(\mathrm{p}<0.05)$. At 15 days, MTA and Biodentine ${ }^{\circledR}$ showed complete hard tissue bridge $(\mathrm{p}<0.05)$. For immunostaining, Biodentine ${ }^{\circledR}$ obtained greater marking than the glass ionomer for Fibronectin and Tenascin. Biodentine ${ }^{\circledR}$, white MTA Angelus ${ }^{\circledR}$ and calcium hydroxide showed the ability to induce mineralization according to the applied methodology. However, Biodentine ${ }^{\circledR}$ showed superior tissue response than glass ionomer and calcium hydroxide.
\end{abstract}

Keywords: Pulpotomy; Inflammation; Materials testing; Endodontics.

\title{
Resumen
}

La pulpotomía es una opción de tratamiento conservador cuya función es preservar la vitalidad pulpar en la porción radicular, con el uso de materiales biocompatibles en este tejido remanente. Este estudio tuvo como objetivo evaluar la respuesta biológica del remanente pulpar a los cementos biocerámicos restauradores Biodentine ${ }^{\circledR}$ y MTA Branco Angelus ${ }^{\circledR}$ en comparación con el hidróxido de calcio después de la pulpotomía. Se utilizaron veinticuatro ratas macho a las que se les expusieron las pulpas coronarias del primer y segundo molares y se les extrajo con una cureta afilada. El tejido pulpar restante recibió uno de los materiales experimentales: Biodentine ${ }^{\circledR}$, MTA Angelus ${ }^{\circledR}$ o Ca $(\mathrm{OH}) 2+$ agua destilada y se selló con ionómero de vidrio. Un grupo se selló directamente con ionómero de vidrio como grupo de control negativo. A los 7 y 15 días, los animales fueron sacrificados y las piezas sometidas a procesamiento histológico para evaluar el proceso inflamatorio colocándolas en HE e inmunohistoquímica (Fibronectina y Tenascina), mediante la atribución de puntajes de 1 a 4. Formación de puentes de tejido duro Se observó en tinción HE, evaluando presencia, continuidad y morfología. Los datos se sometieron a la prueba de Kruskal Wallis y Dunn (p <0,05). El análisis estadístico mostró que a los 7 días el MTA y el Ca $(\mathrm{OH}) 2$ tenían una mayor continuidad del puente de tejido duro que el ionómero de vidrio $(\mathrm{p}<0,05)$. Biodentine ${ }^{\circledR}$ mostró mejores aspectos morfológicos en comparación con el ionómero de vidrio ( $\mathrm{p}<0.05)$. A los 15 días, MTA y Biodentine mostraron un puente de tejido duro completo $(\mathrm{p}<0,05)$. Para la inmunotinción, Biodentine ${ }^{\circledR}$ obtuvo un mayor marcado que el ionómero de vidrio para fibronectina y tenascina. Biodentine $^{\circledR}$, MTA Angelus Branco e hidróxido de calcio mostraron la capacidad de inducir la mineralización según la metodología aplicada. Sin embargo, Biodentine ${ }^{\circledR}$ mostró una mejor respuesta tisular que el ionómero de vidrio y el hidróxido de calcio.

Palabras clave: Pulpotomía; Inflamación; Ensayo de materiales; Endodoncia.

\section{Introdução}

O capeamento pulpar direto ou a pulpotomia são opções de tratamentos conservadores que têm como função preservar a vitalidade pulpar (Parirokh, Torabinejad \& Dummer, 2018). Estes procedimentos são realizados através da adição de um material biocompatível em contato direto com a polpa dentária e como resposta, produz uma barreira mineralizadora. O processo de formação da ponte de tecido duro, denominada dentina terciária reparadora, ocorre através da resposta pulpar frente à um 
material capeador, iniciando um processo inflamatório, que promove a liberação de biomoléculas, como fatores de crescimento (TGFs), proteínas ósseas morfogenéticas (BMPs), interleucinas (IL), fatores de crescimento de fibroblastos (FGFs) e fibronectina (Leites et al., 2011) que atrairão células progenitoras indiferenciadas para se diferenciar em odontoblast-like, também denominadas neo-odontoblastos. Este, por sua vez, tem função semelhante à de odontoblastos, secretar a dentina reparadora para proteger o tecido pulpar de uma contaminação bacteriana (Murray, Teixeira, Oliveira, Jacomini \& Silva, 2003; Tziaras 1994).

No processo de reparação tecidual e formação da barreira mineralizadora, algumas glicoproteínas presentes na matriz extracelular, como a fibronectina (FNC) e da tenascina (TNC) são decisivas (Leites et al., 2011; Piva, Tarquinio, Demarco, Silva $\&$ de Araujo, 2006) A importância destas foram descritas em estudos anteriores a respeito de processos de reparo tecidual (Chiquet-Ehrismann, 1990) e odontogênese (Lesot et al.,1993; Thesleff, Mackie, Vainio \& Chiquet-Ehrismann, 1987). Na cicatrização, a FNC facilita a agregação plaquetária através de sua deposição no colágeno e/ou fibrina (Aukhil, Sahlberg \& Thesleff, 1996). Também atua na angiogênese, pela estimulação da migração de células endoteliais, servindo como guia para o movimento das células epiteliais através do tecido de granulação e auxiliando no reconhecimento da membrana basal subjacente, de tal forma que a ceratinização possa ocorrer de forma normal (Grinnel, 1984; Leite et al., 2021).

Durante o reparo do tecido pulpar, a glicoproteína FNC na presença de um material biocompatível, como o cimento de hidróxido de cálcio, poderia mediar a migração, proliferação e adesão de células que sintetizam uma matriz de fibrodentina rica em FNC (Hung et al., 2014; Yoshiba, Yoshiba, Nakamura, Iwaku \& Ozawa, 1996), induzindo a diferenciação de neoodontoblastos (Leite et al., 2021). A TNC, por sua vez, tem papel estrutural e pode servir como moduladora da função da célula via interações com seus receptores. Ela promove a inibição de contato célula-célula e pode modificar adesividade entre células e FNC, promovendo uma ligação menos resistente entre elas, permitindo a movimentação celular, com a manutenção da integridade de seu citoesqueleto, durante a migração (Chiquet-Ehrismann., 1990; Sage \& Bornstein, 1991; Aukhil et al., 1996; Shrestha et al., 1996).

Alguns fatores são importantes para o sucesso do capeamento pulpar direto, tais como idade do paciente. De acordo com Leites et al. (2011), dentes com polpas mais jovens tem capacidade regenerativa maior, devido a quantidade de células mesenquimais do que polpas menos jovens. Outros fatores como a característica da exposição pulpar (exposição acidental ou cariosa e o seu tamanho), além da intensidade da hemorragia pulpar (Bogen, Kim \& Bakland, 2008) e a escolha do material forrador, também interferem no prognóstico do tratamento (Çaliskan \& Guneri, 2017; Leites et al., 2011; Marques et al., 2015)

A respeito disso, diferentes materiais são utilizados em terapias conservadoras, nas quais a polpa encontra-se com vitalidade para promover a formação da dentina reparadora. O material ideal para ser utilizado na proteção do complexo dentinopulpar deve ser biocompatível (Minamikawa et al., 2011), e um dos mais utilizados para esse propósito é o hidróxido de cálcio $\left[\mathrm{Ca}(\mathrm{OH})_{2}\right]$ (Silva et al., 2019; Yaemkleebbua, Osathanon, Nowwarote, Limjeerajaus \& Sukarawan, 2019; Youssef et al., 2019).

$\mathrm{O} \mathrm{Ca}(\mathrm{OH})_{2}$ é amplamente utilizado quando se trata de agente de proteção pulpar (Paranjpe, Zhang \& Johnson, 2010), pois possui baixo custo, fácil manuseio e estimula formação de tecido duro (Accorinte et al., 2008). No entanto, evidências sugerem que a infiltração e a porosidade podem ocorrer devido a sua elevada solubilidade em água, o que poderia levar ao fracasso do capeamento pulpar (Schuurs, Gruythuysen, Wesselink, 2000; AI-Hezaimi et al., 2011). Além disso, o hidróxido de cálcio destrói uma fina camada do tecido pulpar subjacente, deixando uma camada necrótica, devido ao seu pH elevado (Estrela \& Holland, 2003).

Como alternativa ao $\mathrm{Ca}(\mathrm{OH})_{2}$, foi desenvolvido o agregado de trióxido mineral (MTA), que pode ser empregado em perfurações radiculares e de furca, apicificação, retro obturação, reabsorções radiculares, selamento apical, pulpotomia e capeamento pulpar direto, capaz de estimular a formação de ponte de dentina (Tawil, Duggan \& Galicia, 2015). O MTA é um material bioativo, biocompatível, estável e possui alta capacidade de vedação (Bueno et al., 2019; Faraco \& Holland, 2001). 
Entretanto, é de difícil manipulação, seu tempo de presa é longo, o custo é elevado e pode causar pigmentação do elemento dentário (Dammaschke, Gerth, Zuchner \& Schãfer 2005).

Através de pesquisas acerca do cimento MTA, foi desenvolvido em 2009 um cimento a base de silicato de tricálcico $\left(\mathrm{Ca}_{3} \mathrm{SiO}_{5}\right)$, denominado Biodentine ${ }^{\circledR}$ (Septodont, Saint Maur des Fossés, France), visando melhorar propriedades físicoquímicas. O material consiste em um pó e líquido. O pó contém principalmente silicato tricálcico e dicálcico $\left(3 \mathrm{CaO} \mathrm{SiO}_{2}\right.$ e $2 \mathrm{CaO}$ $\left.\mathrm{SiO}_{2}\right)$, os principais componentes do cimento Portland, bem como carbonato de cálcio $\left(\mathrm{CaCO}_{3}\right)$ e o dióxido de zircónio $\left(\mathrm{ZrO}_{2}\right)$ utilizado para dar contraste ao cimento. O líquido é composto de cloreto de cálcio $\left(\mathrm{CaCl}_{2} 2 \mathrm{H}_{2} \mathrm{O}\right)$, usado como um acelerador de configuração, além de atuar como agente de redução de água em uma solução aquosa em conjunto com o policarboxilato, um agente superplastificante (Chicarelli et al., 2021; Laurent, Camps \& About, 2012). O produto possui propriedades mecânicas semelhantes à dentina, podendo ser utilizado como um substituto na coroa e tem seu uso similar ao do MTA no canal radicular (Koubi et al., 2013; Laurent et al., 2012).

Dessa forma, este trabalho teve como objetivo avaliar a resposta biológica do remanescente pulpar após procedimento de pulpotomia frente à materiais reparadores biocerâmicos, bem como a indução destes na produção de glicoproteínas que estão presentes no processo de reparo tecidual dos materiais Biodentine ${ }^{\circledR}$, MTA Branco Angelus ${ }^{\circledR}, \mathrm{Ca}(\mathrm{OH})_{2}$ por meio da análise histológica e imunoistoquímica das glicoproteínas FNC e TNC em tecido pulpar de ratos wistar.

\section{Metodologia}

\section{Animais}

O cálculo amostral foi baseado em um estudo prévio (Chicarelli et al., 2021) com o programa SigmaPlot 12.0: considerando um poder de análise de 0.80 , diferença mínima entre as médias de 0,22 e desvio padrão (SD) do erro médio de 0.11 , garantiu um mínimo de seis dentes $(\mathrm{n}=6)$ por grupo experimental.

Vinte e quatro ratos Wistar machos, pesando 250-280g, foram utilizados neste estudo. Os animais foram alojados em gabinetes com temperatura controlada e com água e comida ad libitum. O estudo foi realizado de acordo com as normas da Comissão de Ética da Faculdade de Odontologia de Araçatuba - UNESP, Processo nº 2014-00452.

\section{Pulpotomia}

Os animais foram igualmente divididos em 4 grupos experimentais: Biodentine ${ }^{\circledR}$, MTA Angelus Branco ${ }^{\circledR}$, $\mathrm{Ca}(\mathrm{OH})_{2}+$ água destilada e um grupo sem capeamento e selado diretamente com Ionômero de vidro (Fuji IX, GC, Tóquio, Japão). Cada grupo experimental $(\mathrm{n}=6)$ foi dividido e analisado em períodos de tempo 7 e 15 dias, resultando em 3 animais em cada período. Em cada animal foram utilizados os 2 primeiros molares inferiores (direito e esquerdo). Os animais foram anestesiados com cloridrato de Xilanzina $2 \%(25 \mathrm{mg} / \mathrm{kg})$ e cloridrato de Ketamina $10 \%(50 \mathrm{mg} / \mathrm{kg})$, via intramuscular. Previamente ao acesso coronário foi realizada a desinfecção dos primeiros molares com gluconato de clorexidina $0,2 \%$.

Com auxílio de um microscópio, o acesso à câmara pulpar foi realizado com broca carbide $1 / 2 \mathrm{em}$ alta rotação, seguida da remoção do teto da câmara pulpar, a pulpotomia foi realizada com uma cureta modificada. A irrigação foi realizada com solução de soro fisiológico estéril. O sangramento foi controlado com irrigação salina, usando bolinhas de algodão estéreis. O tecido pulpar remanescente recebeu um dos materiais: Biodentine ${ }^{\circledR}$, MTA Angelus ${ }^{\circledR}, \mathrm{Ca}(\mathrm{OH})_{2}+$ água destilada e selados com ionômero de vidro. O grupo controle foi deixado sem material capeador, selado diretamente com ionômero de vidro sobre o remanescente pulpar.

Após os períodos de 7 e 15 dias, os animais foram eutanasiados por overdose anestésica, com injeção intramuscular Xilazina/Quetamina. A pele que corresponde à face do animal foi removida e cortes realizados com uma tesoura no ângulo da boca, permitiram a separação da mandíbula do resto do crânio do animal, e em seguida a separação da mandíbula esquerda e 
direita. Com as mandíbulas separadas as peças foram reduzidas e outro corte foi realizado tangente à superfície distal dos terceiros molares. As mandíbulas foram imediatamente colocadas em frascos individuais contendo solução de formol a $10 \%$ tamponada por 24 horas, e na sequência lavadas por 12 horas e descalcificação em EDTA por aproximadamente 30 dias.

Para o processamento histológico e imunohistoquímico, as mandíbulas foram desidratadas, incluídas em parafina e cortadas em micrótomo ( $5 \mu \mathrm{m}$ de espessura) de forma que fosse possível visualizar a câmara pulpar. A lâminas para análise de tecido duro, reação inflamatória e partículas do material capeador foram coradas com Hematoxilina e Eosina (HE) e para as lâminas para imunomarcação foi utilizado reagentes para FN e TN (Santa Cruz, Biotechnology, CA, USA).

As lâminas coradas em HE foram analisadas seguindo critérios modificados de Faraco \& Holland. (2001) (Faraco \& Holland, 2001): análise da ponte de tecido duro (continuidade, aspectos morfológicos), reação inflamatória (número de células e extensão da reação), partículas do material capeador. Todos esses eventos histomorfológicos foram avaliados pelos escores 1 a 4, sendo 1 o melhor resultado e 4 o pior. A imunomarcação seguiu os critérios de escores: 0 = ausência de imunomarcação; 1 = baixo padrão de imunomarcação; 2 = moderado padrão de imunomarcação; 3 = alto padrão de imunomarcação; 4 = padrão extremamente alto de imunomarcação. Os dados foram analisados estatisticamente no programa SigmaPlot V12.0 (Systat Software, San Jose, USA) pelo teste de Kruskal-Wallis e Dunn; O valor de $\mathrm{P}<0,05$ foi considerado significativo.

\section{Resultados}

\section{Avaliação inflamatória}

\section{Ionômero de vidro}

Aos 7 e 15 dias observou-se ausência da ponte de tecido duro, grande infiltrado inflamatório e presença de linfócitos e neutrófilos (Figura 1- A, B).

\section{Biodentine $^{\circledR}$}

Aos 7 dias observou-se presença da ponte de tecido duro com pequena solução de continuidade com o material capeador apresentando calcificações irregulares nas paredes laterais da cavidade com sinais tênues de calcificação, grande infiltrado inflamatório e presença de linfócitos, neutrófilos e macrófagos. O Biodentine ${ }^{\circledR}$ apresentou melhores aspectos morfológicos que o ionômero de vidro $(\mathrm{p}<0,05)$ (Tabela 1). A extensão desse processo inflamatório foi pequena com células nas imediações do material capeador e ausência de partículas de material capeador na polpa (Figura 1C). Aos 15 dias observou-se presença completa de ponte de tecido duro (Tabela 1) com áreas de dentina isolada ou associada à ponte de tecido duro, houve redução na intensidade da inflamação com presença de fibroblastos e vasos sanguíneos. Não foi observada presença de partículas de material capeador na polpa (Figura 1D).

\section{Hidróxido de Cálcio}

Aos 7 e 15 dias observou-se a presença da ponte de tecido duro com pequena solução de continuidade comunicando a polpa dental com o material capeador, com calcificações irregulares e grande infiltrado inflamatório. O hidróxido de cálcio obteve maior continuidade da ponte de tecido duro que o ionômero de vidro ( $\mathrm{p}<0.05)$ (Tabela 1) (Figura 1- E, F).

\section{MTA Angelus Branco ${ }^{\circledR}$}

Aos 7 dias o MTA mostrou continuidade da ponte de tecido duro ( $\mathrm{p}<0.05)$ (Tabela 1). Aos 15 dias observou-se presença completa de ponte de tecido duro (Tabela 1) com áreas de dentina isolada ou associada à ponte de tecido duro, houve redução na intensidade da inflamação, e não foi observado presença de partículas de material capeador na polpa (Figura 1H). 
Figura 1. (HE - 100X). Imagens representativas da reação inflamatória dos grupos experimentais e ionômero de vidro. Aos 7 dias, os grupos Ionômero de vidro (A), Biodentine $(\mathrm{C}), \mathrm{Ca}(\mathrm{OH})_{2}(\mathrm{E})$ e MTA $(\mathrm{G})$ apresentaram infiltrado inflamatório de moderado a severo. O Biodentine $(\mathrm{C}), \mathrm{Ca}(\mathrm{OH})_{2}(\mathrm{E})$ e MTA $(\mathrm{G})$ induziram formação de ponte de tecido duro. Aos 15 dias, os grupos Biodentine (D), e MTA (H) apresentaram redução no infiltrado inflamatório e aumentaram a continuidade da ponte de tecido duro (setas amarelas).

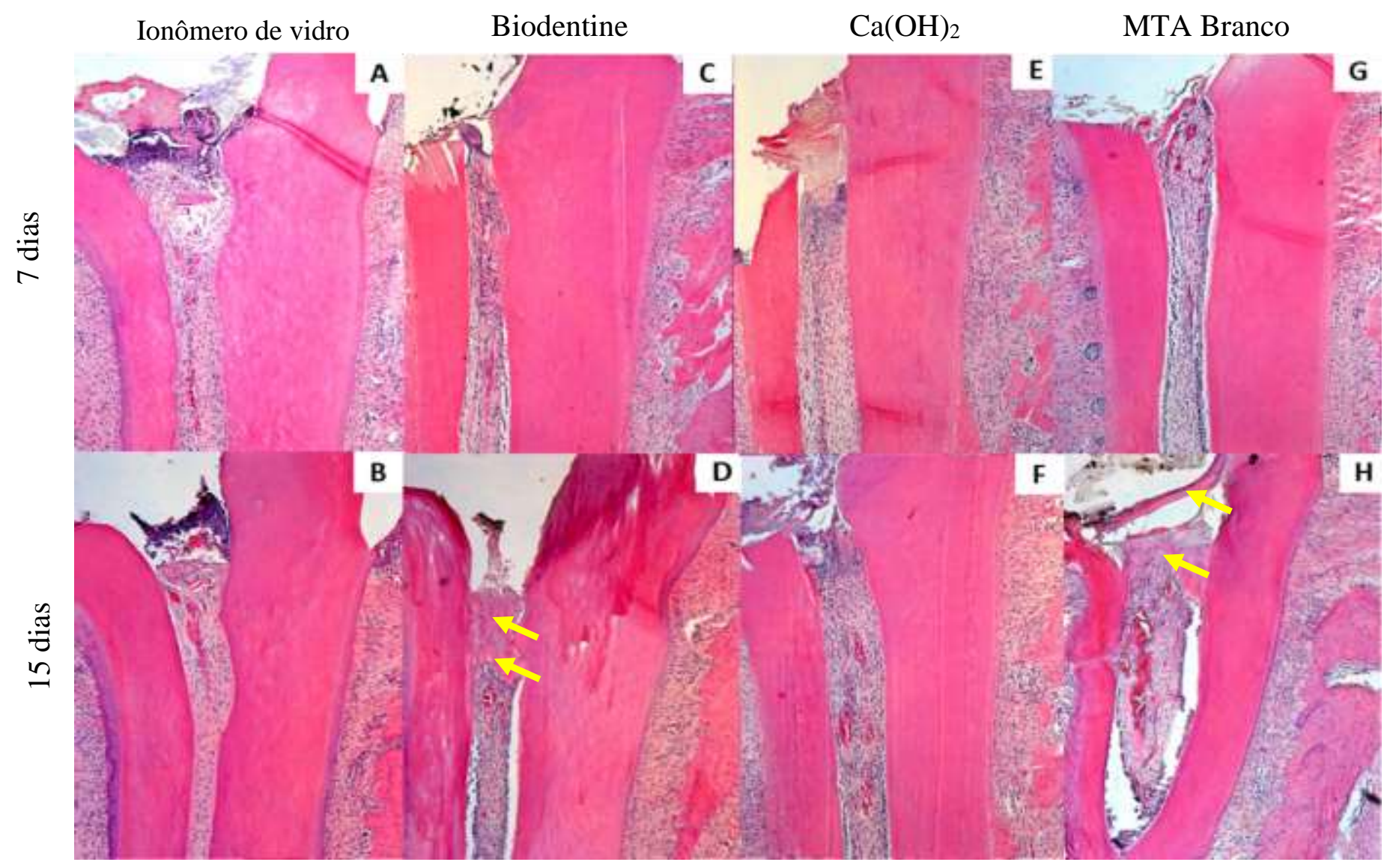

Fonte: Autores. 
Tabela 1. Mediana dos espécimes de acordo com escores para pulpotomia.

\begin{tabular}{|c|c|c|c|c|c|c|c|c|c|c|}
\hline BIODENTINE & \multicolumn{5}{|c|}{7 Dias } & \multicolumn{5}{|c|}{15 Dias } \\
\hline Continuidade & 3 & 2 & 3 & 2 & 2 & 1 & 1 & 1 & 2 & 1 \\
\hline Morfologia & 3 & 1 & 3 & 1 & 1 & 1 & 1 & 1 & 1 & 1 \\
\hline Intensidade da inflamação & 4 & 4 & 4 & 4 & 4 & 1 & 3 & 1 & 4 & 1 \\
\hline Extensão da inflamação & 3 & 2 & 2 & 2 & 2 & 1 & 2 & 1 & 2 & 1 \\
\hline Estado geral polpa & 2 & 2 & 2 & 2 & 2 & 1 & 2 & 1 & 2 & 1 \\
\hline Partículas de material capeador & 1 & 1 & 1 & 1 & 1 & 1 & 1 & 1 & 1 & 1 \\
\hline $\mathrm{Ca}(\mathrm{OH}) 2$ & \multicolumn{5}{|c|}{7 Dias } & \multicolumn{5}{|c|}{15 Dias } \\
\hline Continuidade & 2 & 2 & 2 & 2 & 3 & 2 & 2 & 2 & 2 & 2 \\
\hline Morfologia & 2 & 2 & 1 & 3 & 3 & 2 & 2 & 2 & 2 & 2 \\
\hline Intensidade da inflamação & 4 & 4 & 4 & 3 & 4 & 4 & 4 & 4 & 4 & 4 \\
\hline Extensão da inflamação & 3 & 3 & 2 & 2 & 3 & 2 & 2 & 2 & 2 & 2 \\
\hline Estado geral polpa & 2 & 2 & 2 & 2 & 2 & 2 & 2 & 2 & 2 & 2 \\
\hline Partículas de material capeador & 1 & 1 & 1 & 1 & 1 & 1 & 1 & 1 & 1 & 1 \\
\hline IONÔMERO DE VIDRO & \multicolumn{5}{|c|}{7 Dias } & \multicolumn{5}{|c|}{15 Dias } \\
\hline Continuidade & 4 & 4 & 4 & 4 & 4 & 4 & 4 & 4 & 4 & 4 \\
\hline Morfologia & 4 & 4 & 4 & 4 & 4 & 4 & 4 & 4 & 4 & 4 \\
\hline Intensidade da inflamação & 4 & 4 & 4 & 3 & 3 & 4 & 4 & 4 & 4 & 4 \\
\hline Extensão da inflamação & 2 & 2 & 3 & 2 & 2 & 2 & 3 & 2 & 3 & 2 \\
\hline Estado geral polpa & 2 & 2 & 2 & 2 & 2 & 2 & 2 & 3 & 2 & 2 \\
\hline Partículas de material capeador & 1 & 1 & 2 & 1 & 2 & 1 & 1 & 1 & 1 & 1 \\
\hline $\begin{array}{r}\text { MTA BRANCO } \\
\end{array}$ & \multicolumn{5}{|c|}{7 Dias } & \multicolumn{5}{|c|}{15 Dias } \\
\hline Continuidade & 3 & 2 & 2 & 2 & 2 & 1 & 1 & 1 & 1 & 1 \\
\hline Morfologia & 3 & 2 & 2 & 2 & 2 & 1 & 1 & 1 & 1 & 1 \\
\hline Intensidade da inflamação & 3 & 3 & 4 & 4 & 2 & 2 & 1 & 2 & 2 & 2 \\
\hline Extensão da inflamação & 2 & 2 & 3 & 2 & 2 & 2 & 1 & 1 & 1 & 2 \\
\hline Estado geral polpa & 2 & 2 & 2 & 2 & 1 & 2 & 1 & 2 & 2 & 2 \\
\hline Partículas de material capeador & 1 & 1 & 1 & 1 & 1 & 1 & 1 & 1 & 1 & 1 \\
\hline
\end{tabular}

Fonte: Autores.

\section{Avaliação Imunoistoquímica}

\section{Fibronectina}

Ao se avaliar a imunomarcação da glicoproteína fibronectina houve maior imunomarcação para o Biodentine ${ }^{\circledR}$, e o hidróxido de cálcio em relação ao ionômero de vidro aos 7 dias (p<0,05). Aos 15 dias o padrão de imunomarcação foi mais intenso nos grupos Biodentine ${ }^{\circledR}$, Hidróxido de cálcio e MTA Branco Angelus (Figura 2- F, J, N) (Tabela 2).

\section{Tenascina}

Ao se avaliar a imunomarcação da Tenascina foi observado que aos 7 dias o Biodentine ${ }^{\circledR}$ apresentou maior padrão de imunomarcação em relação aos demais grupos (p<0,05) (Figura 2 - G) (Tabela 3). Já aos 15 dias o Biodentine ${ }^{\circledR}$, Hidróxido de cálcio e MTA Branco Angelus apresentaram um padrão de imunomarcação maior que o grupo ionômero de vidro $(\mathrm{p}<0,05)$ (Figura 2 H, L, P). 
Figura 2. Grupos: Ionômero de vidro (A-D), Biodentine ${ }^{\circledR}(\mathrm{E}-\mathrm{H}), \mathrm{Ca}(\mathrm{OH})_{2}$ (I-L) e MTA Angelus Branco ${ }^{\circledR}(\mathrm{M}-\mathrm{P})$. Tempos de análise: Fibronectina 7 dias (A, E, I, M), Fibronectina 15 dias (B, F, J, N), Tenascina 7 dias (C, G, K, O), Tenascina 15 dias (D, H, L, P). [Marcação imunoistoquímica para Fibronectina/ Tenascina; 250X].

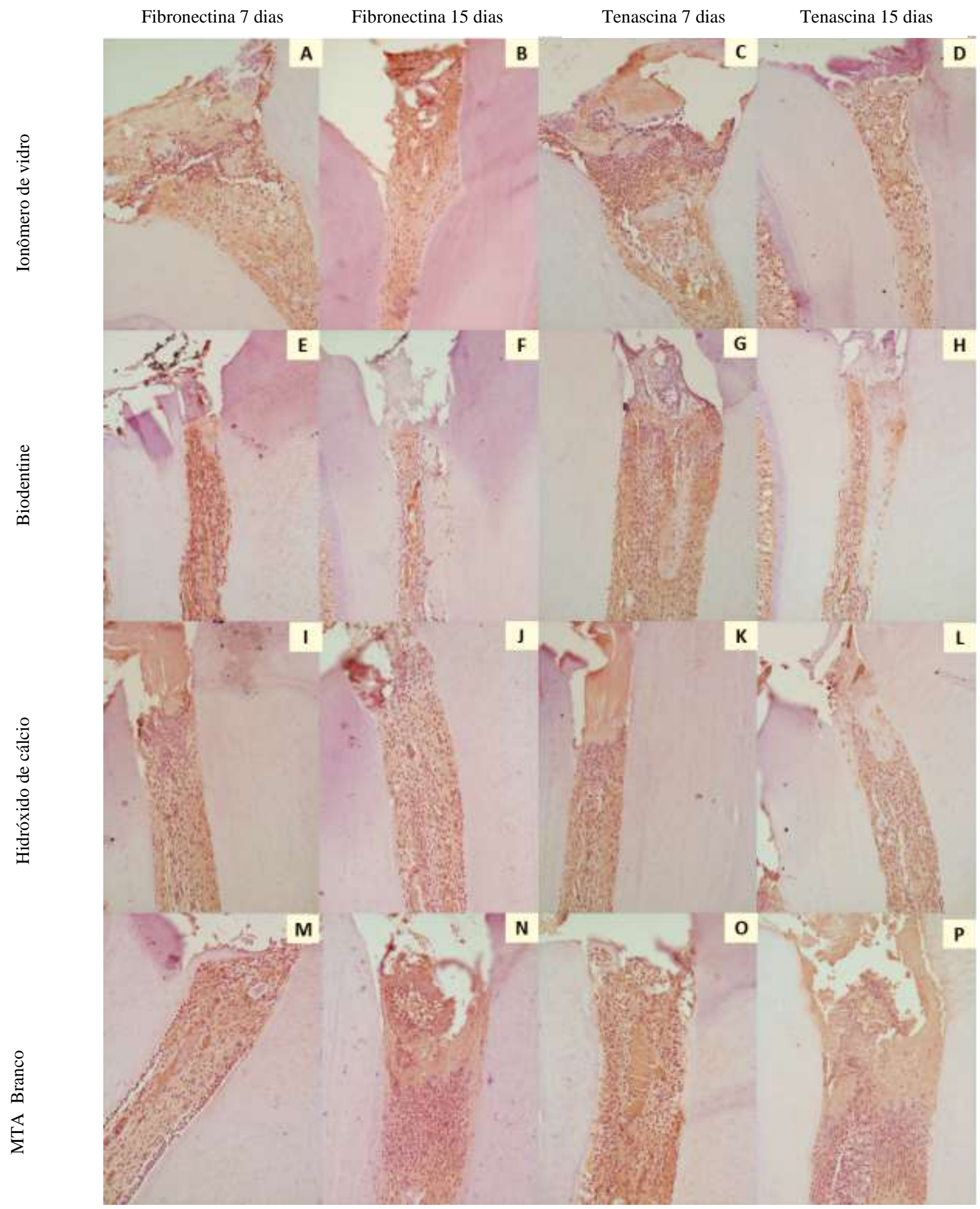

Fonte: Autores. 
Tabela 2. Mediana dos espécimes de acordo com escores para Fibronectina.

\begin{tabular}{|c|c|c|c|c|c|c|c|}
\hline \multirow[t]{2}{*}{ Tempo } & \multirow{2}{*}{ Material } & \multicolumn{4}{|c|}{ Escores para FNC } & \multirow{2}{*}{ Mediana } & \multirow[b]{2}{*}{ Valor de $\mathrm{p}$} \\
\hline & & 1 & 2 & 3 & 4 & & \\
\hline \multirow[t]{4}{*}{7 dias } & Controle & $8 / 8$ & 0 & 0 & 0 & 1 & \multirow{4}{*}{$\mathrm{P}=<0.001$} \\
\hline & MTA Branco & $6 / 8$ & $2 / 8$ & 0 & 0 & 1 & \\
\hline & Biodentine & $2 / 8$ & $6 / 8$ & 0 & 0 & 2 & \\
\hline & $\mathrm{Ca}(\mathrm{OH})_{2}$ & $1 / 8$ & $7 / 8$ & 0 & 0 & 2 & \\
\hline \multirow[t]{4}{*}{15 dias } & Controle & $8 / 8$ & 0 & 0 & 0 & 1 & \multirow{4}{*}{$\mathrm{P}=<0.001$} \\
\hline & MTA Branco & 0 & $8 / 8$ & 0 & 0 & 2 & \\
\hline & Biodentine & 0 & $8 / 8$ & 0 & 0 & 2 & \\
\hline & $\mathrm{Ca}(\mathrm{OH})_{2}$ & 0 & $8 / 8$ & 0 & 0 & 2 & \\
\hline
\end{tabular}

Fonte: Autores.

Tabela 3. Mediana dos espécimes de acordo com escores para Tenascina.

\begin{tabular}{|c|c|c|c|c|c|c|c|}
\hline \multirow[t]{2}{*}{ Tempo } & \multirow{2}{*}{ Material } & \multicolumn{4}{|c|}{ Escores para TNC } & \multirow{2}{*}{ Mediana } & \multirow[b]{2}{*}{ Valor de $\mathrm{p}$} \\
\hline & & 1 & 2 & 3 & 4 & & \\
\hline \multirow[t]{4}{*}{7 dias } & Controle & $8 / 8$ & 0 & 0 & 0 & 1 & \multirow{4}{*}{$\mathrm{P}=0.015$} \\
\hline & MTA Branco & $6 / 8$ & $2 / 8$ & 0 & 0 & 1 & \\
\hline & Biodentine & 0 & $8 / 8$ & 0 & 0 & 2 & \\
\hline & $\mathrm{Ca}(\mathrm{OH})_{2}$ & $7 / 8$ & $1 / 8$ & 0 & 0 & 1 & \\
\hline \multirow[t]{4}{*}{15 dias } & Controle & $8 / 8$ & 0 & 0 & 0 & 1 & \multirow{4}{*}{$\mathrm{P}=<0.001$} \\
\hline & MTA Branco & 0 & $8 / 8$ & 0 & 0 & 2 & \\
\hline & Biodentine & 0 & $6 / 8$ & $2 / 8$ & 0 & 2 & \\
\hline & $\mathrm{Ca}(\mathrm{OH})_{2}$ & 0 & $8 / 8$ & 0 & 0 & 2 & \\
\hline
\end{tabular}

\section{Discussão}

Fonte: Autores.

O presente trabalho objetivou avaliar a resposta biológica de cimentos biocerâmicos reparadores após pulpotomia em ratos wistar, analisando o perfil inflamatório e a imunomarcação de fibronectina e tenascina, presentes no processo de reparo tecidual.

O modelo animal tem sido vastamente utilizado em pesquisas odontológicas, seja em cães (Dezan Junior et al., 2021), preás (Lacativa, Loyola \& Sousa, 2012) ou ratos (Bueno et al., 2016; Bueno et al., 2019), entre outros, podendo ainda variar em animais sistemicamente comprometidos, como ratos hipertensos (Martins, Sasaki, Hirai, Andrada \& Gomes-Filho, 2016) ou diabéticos (Bueno et al., 2021). O modelo utilizado para este estudo foi de pulpotomia em molares de ratos wistar, previamente utilizado na literatura (Esmeraldo, Carvalho, Carvalho, Lia \& Costa, 2013; Lima et al., 2011; Minic et al., 2021). De acordo com Dammaschke et al. (2005), os molares de ratos possuem tecido pulpar anatomicamente, histologicamente e fisiologicamente semelhantes aos molares humanos (Dammaschke et al., 2005).

No que diz respeito à análise histológica, o infiltrado inflamatório pôde ser analisado pela coloração de HE, uma vez que possibilita a visualização das células inflamatórias presentes no local e quantificar essa inflamação, corroborando a metodologia de estudos prévios (Benetti et al., 2021; Bueno et al., 2019). Embora colorações como Von Kossa sejam indicados para avaliação de formação de tecido mineralizado, a metodologia em dentes inviabiliza tal análise, uma vez que os dentes passam por processo de desmineralização durante processamento laboratorial para serem cortados em micrótomo. Portanto, indícios de mineralização sob coloração de HE foram observados, baseado em pesquisas anteriores (Borlina et al., 2010; Faraco \& Holland, 2001). 
As glicoproteínas usadas nessa pesquisa foram fibronectina (FNC) e tenascina (TNC). De acordo com Tziafas et al. (1995), uma matriz rica em FNC pode servir como um reservatório de fatores de crescimento e moléculas que sinalizam para a diferenciação de odontoblastos em dentina terciária. Já a TNC pode facilitar a adesão entre células e a FNC, facilitando a migração celular através da matriz, associada com reparo tecidual (Benetti et al., 2021; Piva et al., 2006). A expressão de TNC pode ainda estar ligada ao processo de biomineralização (Benetti et al., 2021; Piva et al., 2006; Zarrabi et al., 2011).

O uso das glicoproteínas fibronectina e tenascina para avaliar resposta pulpar a materiais endodônticos foi previamente utilizada (Baldissera et al., 2013; Moradi, Saghravanian Moushekhian, Fatemi \& Forghani, 2015; Zarrabi et al., 2011), embasando nossa metodologia. Estudos investigando a expressão de TN e FN após capeamento pulpar com hidróxido de cálcio ou MTA em dentes humanos ou suínos sugeriram que a diferenciação de odontoblastos poderia ser regulada pela expressão dessas duas glicoproteínas (Leites et al., 2011; Piva et al., 2006). Recentemente, uso de doses de fibronectina com hidrogel foi relatada na técnica de regeneração tecidual guiada para regeneração pulpar em dentes com formação radicular incompleta (Leite et al., 2021).

As glicoproteínas fibronectina e tenascina mostraram maior padrão de imunomarcação aos 7 dias comparados ao ionômero de vidro $(\mathrm{p}<0.05)$. A resposta do presente estudo foi semelhante ao trabalho de Leites et al. (2011) em relação ao padrão de imunomarcação das glicoproteínas FNC e TNC dos grupos MTA e Hidróxido de Cálcio.

Um estudo recente utilizando o Biodentine em pulpotomia após indução de pulpite em ratos wistar mostrou potencial deposição de tecido mineralizado no local da lesão pulpar e elevado reparo tecidual (Minic et al., 2021), corroborando nossos resultados no qual biocerâmicos reparadores como Biodentine e MTA mostraram elevado padrão de imunomarcação das glicoproteínas FNC e TNC envolvidas com reparo tecidual.

Nosso estudo demonstrou a biocompatibilidade dos cimentos biocerâmicos reparadores após pulpotomia. Tal resultado corrobora um estudo recente em subcutâneo de ratos wistar (Valentim et al., 2021) mostrando a baixa reposta inflamatória após 15, 30 e 60 dias do Biodentine e MTA. Estudos anteriores já haviam demonstraram a biocompatibilidade do MTA Angelus (Benetti et al. 2021; Bueno et al., 2019; Cosme-Silva et al., 2019a; Cosme-Silva et al., 2019b) confirmando este como um padrão ouro (Torabinejad, Parirokh, \& Dummer, 2018). Um estudo clínico avaliando os mesmos cimentos Biodentine ${ }^{\circledR}$ e MTA na pulpotomia, após 12 meses de acompanhamento, relatou taxas de sucesso dos materiais em 100\% e 97.4\% e as taxas de sucesso radiográfico de $94.9 \%$ e $97.4 \%$, respectivamente (Cuadros-Fernadez et al., 2016). Outro estudo de acompanhamento clínico após pulpotomia em dentes decíduos por um período de 24 meses utilizando Biodentine e MTA, mostrou que as taxas de sucesso foram de $100 \%$ para o grupo Biodentine e 89,4\% para o MTA, sem diferenças estatisticamente significantes (Çelik et al., 2019).

Um estudo prévio conduzido por De Rossi et al. (2014) analisou histologicamente e radiograficamente a ação do Biodentine e do MTA. Na análise radiográfica, o Biodentine promoveu maior mineralização em dentes de cães que o MTA. Entretanto, na análise histológica, ambos os materiais avaliados apresentaram comportamento semelhante. Tal resultado está em acordo com nossos achados, uma vez que na análise histológica (HE), o Biodentine não mostrou diferenças significativas nas análises de formação de tecido duro e inflamação nos períodos avaliados em relação ao MTA.

O Biodentine e o MTA no período de 15 dias, apresentaram melhores resultados quando comparados ao ionômero de vidro em relação a continuidade e morfologia da ponte de tecido duro $(\mathrm{p}<0.05)$. Resultados semelhantes foram relatados por Nowicka et al. (2015) avaliando o volume e continuidade de mineralização formada pelo Biodentine, MTA, Ca(OH) 2 e o Single Bond em dentes humanos, após um período de 45 dias, onde a ponte de dentina mais homogênea e com maior volume foi promovida pelo Biodentine e MTA, sem diferenças significativas em relação ao $\mathrm{Ca}(\mathrm{OH})_{2}$ e com diferenças em relação ao Single Bond Universal.

Pesquisas anteriores investigando o MTA Cinza e o MTA Branco afirmaram que a ponte de tecido duro estará completamente formada a partir de 28 dias (Holan, Eidelman \& Fuks, 2005; Salako et al., 2003). Em contrapartida, no presente 
estudo, aos 7 dias notou-se o início da formação de tecido duro e no período de 15 dias a formação encontrava-se completa. Resultados semelhantes foram encontrados no estudo de Shayegan et al. (2012), que no sétimo dia iniciou-se a formação da ponte de dentina terciária.

No presente estudo, observou-se que os materiais Biodentine ${ }^{\circledast}$, MTA Angelus Branco e o Hidróxido de Cálcio apresentaram capacidade de induzir mineralização na metodologia aplicada, sendo que o Biodentine mostrou melhor resposta tecidual e formação de ponte de tecido duro em comparação com o hidróxido de cálcio e o MTA. Nossos resultados corroboram achados recentes (Abo El-Mal et al., 2021), onde o MTA e o hidróxido de cálcio, quando usados como materiais capeadores, formaram ponte de tecido duro em quase $80 \%$ das amostras.

Recentemente, Chicarelli et al. (2021) demonstraram resultados semelhantes ao do presente estudo, no qual avaliaram a resposta pulpar, a continuidade e a morfologia de barreira mineralizada após capeamento pulpar direto em ratos, comparando os grupos MTA, Biodentine ${ }^{\circledR}$ e hidróxido de cálcio. De acordo com os autores, MTA e Biodentine ${ }^{\circledR}$ promoveram menor reação inflamatória do que o hidróxido de cálcio, assim como induziram formação de barreira mineralizada, mais contínua e uniforme, em acordo com os nossos resultados.

Ainda em conformidade com os resultados observados nesta pesquisa, Cushley et al. (2021) relataram através de uma revisão sistemática que evidências de baixa qualidade sugerem uma alta taxa de sucesso para capeamento pulpar direto em dentes permanentes com polpas expostas por processo carioso, com melhores resultados em longo prazo para MTA e Biodentine em comparação com hidróxido de cálcio.

\section{Conclusão}

Os cimentos biocerâmicos reparadores Biodentine ${ }^{\circledR}$ e MTA Branco Angelus mostraram biocompatibilidade e induziram a imunomarcação de tenascina e fibronectina após pulpotmia, podendo ser indicado para os casos em que ocorre exposição pulpar. Mais estudos com outros imunomarcadores de biomineralização após pulpotomia são encorajados para fornecer dados adicionais e reforçar aplicabilidade clínica.

\section{Agradecimentos}

Este estudo teve apoio financeiro FAPESP, Processo 2014/02327-0.

\section{Referências}

Abo El-Mal, E. O., Abu-Seida, A. M., \& El Ashry, S. H. (2021). Biological evaluation of hesperidin for direct pulp capping in dogs' teeth. International journal of experimental pathology, 102(1), 32-44.

Accorinte, M., Holland, R., Reis, A., Bortoluzzi, M. C., Murata, S. S., Dezan, E., Jr, Souza, V., \& Alessandro, L. D. (2008). Evaluation of mineral trioxide aggregate and calcium hydroxide cement as pulp-capping agents in human teeth. Journal of endodontics, 34(1), 1-6.

Al-Hezaimi, K., Al-Tayar, B. A., Bajuaifer, Y. S., Salameh, Z., Al-Fouzan, K., \& Tay, F. R. (2011). A hybrid approach to direct pulp capping by using emdogain with a capping material. Journal of endodontics, 37(5), 667-672.

Aukhil, I., Sahlberg, C., \& Thesleff, I. (1996). Basal layer of epithelium expresses tenascin mRNA during healing of incisional skin wounds. Journal of periodontal research, 31(2), 105-112.

Baldissera, E. Z., Silva, A. F., Gomes, A. P., Etges, A., Botero, T., Demarco, F. F., \& Tarquinio, S. B. (2013). Tenascin and fibronectin expression after pulp capping with different hemostatic agents: a preliminary study. Brazilian dental journal, 24(3), 188-193.

Benetti, F., Gomes-Filho, J. E., de Azevedo-Queiroz, I. O., Carminatti, M., Conti, L. C., Dos Reis-Prado, A. H., de Oliveira, S., Ervolino, E., Dezan-Júnior, E., \& Cintra, L. (2021). Biological assessment of a new ready-to-use hydraulic sealer. Restorative dentistry \& endodontics, $46(2)$, e21.

Bogen, G., Kim, J. S., \& Bakland, L. K. (2008). Direct pulp capping with mineral trioxide aggregate. Journal of the American Dental Association, 193(3), 305315. 
Borlina, S. C., de Souza, V., Holland, R., Murata, S. S., Gomes-Filho, J. E., Dezan Junior, E., Marion, J. J., \& Neto, D. (2010). Influence of apical foramen widening and sealer on the healing of chronic periapical lesions induced in dogs' teeth. Oral surgery, oral medicine, oral pathology, oral radiology, and endodontics, 109(6), 932-940.

Bueno, C. R. E., Sumida, D. H., Duarte, M. A. H., Ordinola-Zapata, R., Azuma, M. M., Guimarães, G., Pinheiro, T. N., \& Cintra, L. T. A. (2021). Accuracy of radiographic pixel linear analysis in detecting bone loss in periodontal disease: Study in diabetic rats. Saudi Dental Journal, in press, 1-10.

Bueno, C. R. E., Vasques, A. M. V., Cury, M. T. S., Sivieri-Araújo, G., Jacinto, R. C., Gomes-Filho, J. E., Cintra, L., \& Dezan-Júnior, E. (2019). Biocompatibility and biomineralization assessment of mineral trioxide aggregate flow. Clinical oral investigations, 23(1), 169-177.

Bueno, C. R.E., Valentim, D., Marques, V. A., Gomes-Filho, J. E., Cintra, L. T., Jacinto, R. C., \& Dezan-Junior, E. (2016). Biocompatibility and biomineralization assessment of bioceramic-, epoxy-, and calcium hydroxide-based sealers. Brazilian oral research, 30(1), S1806-83242016000100267.

Çalışkan, M. K., \& Güneri, P. (2017). Prognostic factors in direct pulp capping with mineral trioxide aggregate or calcium hydroxide: 2- to 6-year followup. Clinical oral investigations, 21(1), 357-367.

Çelik, B. N., Mutluay, M. S., Arıkan, V., \& Sarı, Ş. (2019). The evaluation of MTA and Biodentine as a pulpotomy materials for carious exposures in primary teeth. Clinical Oral Investigations, 23(2), 661-666.

Chicarelli, L., Webber, M., Amorim, J., Rangel, A., Camilotti, V., Sinhoreti, M., \& Mendonça, M. J. (2021). Effect of Tricalcium Silicate on Direct Pulp Capping: Experimental Study in Rats. European journal of dentistry, 15(1), 101-108.

Chiquet-Ehrismann R. (1990). What distinguishes tenascin from fibronectin? FASEB journal: official publication of the Federation of American Societies for Experimental Biology, 4(9), 2598-2604.

Cosme-Silva, L., Gomes-Filho, J. E., Benetti, F., Dal-Fabbro, R., Sakai, V. T., Cintra, L., Ervolino, E., \& Viola, N. V. (2019a). Biocompatibility and immunohistochemical evaluation of a new calcium silicate-based cement, Bio-C Pulpo. International endodontic journal, 52(5), 689-700.

Cosme-Silva, L., Dal-Fabbro, R., Gonçalves, L. O., Prado, A., Plazza, F. A., Viola, N. V., Cintra, L., \& Gomes Filho, J. E. (2019b). Hypertension affects the biocompatibility and biomineralization of MTA, High-plasticity MTA, and Biodentine®. Brazilian oral research, 33, e060.

Cuadros-Fernández, C., Lorente Rodríguez, A. I., Sáez-Martínez, S. et al. (2016). Short-term treatment outcome of pulpotomies in primary molars using mineral trioxide aggregate and Biodentine: a randomized clinical trial. Clinical Oral Investigation, 20(7), 1639-1645.

Cushley, S., Duncan, H. F., Lappin, M. J., Chua, P., Elamin, A. D., Clarke, M., \& El-Karim, I. A. (2021). Efficacy of direct pulp capping for management of cariously exposed pulps in permanent teeth: a systematic review and meta-analysis. International endodontic journal, 54(4), 556-571.

Dammaschke, T., Gerth, H. U., Züchner, H., \& Schäfer, E. (2005). Chemical and physical surface and bulk material characterization of white ProRoot MTA and two Portland cements. Dental materials: official publication of the Academy of Dental Materials, 21(8), 731-738.

De Rossi, A., Silva, L. A., Gatón-Hernández, P., Sousa-Neto, M. D., Nelson-Filho, P., Silva, R. A., \& de Queiroz, A. M. (2014). Comparison of pulpal responses to pulpotomy and pulp capping with biodentine and mineral trioxide aggregate in dogs. Journal of endodontics, 40(9), 1362-1369.

Dezan-Júnior, E., Bueno, C. R. E., Vasques, A. M. V., Souza, V., de Nery, M. J., Otoboni Filho, J. A., Bernabé, P. F. E., Gomes-Filho, J. E., Cintra, L. T. A., Jacinto, R. C., Sivieri-Araújo, G., \& Holland, R. (2021). Influence of different obturation techniques in coronal bacterial infiltration: study in dogs. Research, Society and Development, 10(4), e11010413884.

Esmeraldo, M. R., Carvalho, M. G., Carvalho, R. A., Lima, R., \& Costa, E. M. (2013). Inflammatory effect of green propolis on dental pulp in rats. Brazilian oral research, 27(5), 417-422.

Estrela, C., \& Holland, R. (2003). Calcium hydroxide: study based on scientific evidences. Journal of applied oral science: revista FOB, 11(4), $269-282$.

Faraco, I. M. Jr \& Holland, R. (2001). Response of the pulp of dogs to capping with mineral trioxide aggregate or a calcium hydroxide cement. Dental traumatology : official publication of International Association for Dental Traumatology, 17(4), 163-166.

Grinnell F. (1984). Fibronectin and wound healing. Journal of cellular biochemistry, 26(2), 107-116.

Holan, G., Eidelman, E., \& Fuks, A. B. (2005). Long-term evaluation of pulpotomy in primary molars using mineral trioxide aggregate or formocresol. Pediatric dentistry, 27(2), 129-136.

Hung, C. J., Hsu, H. I., Lin, C. C., Huang, T. H., Wu, B. C., Kao, C. T., \& Shie, M. Y. (2014). The role of integrin $\alpha$ v in proliferation and differentiation of human dental pulp cell response to calcium silicate cement. Journal of endodontics, 40(11), 1802-1809.

Koubi, G., Colon, P., Franquin, J. C., Hartmann, A., Richard, G., Faure, M. O., \& Lambert, G. (2013). Clinical evaluation of the performance and safety of a new dentine substitute, Biodentine, in the restoration of posterior teeth - a prospective study. Clinical oral investigations, 17(1), $243-249$.

Lacativa, A. M., Loyola, A. M., \& Sousa, C. J. (2012). Histological evaluation of bone response to pediatric endodontic pastes: an experimental study in guinea pig. Brazilian dental journal, 23(6), 635-644.

Laurent, P., Camps, J., \& About, I. (2012). Biodentine(TM) induces TGF- $\beta 1$ release from human pulp cells and early dental pulp mineralization. International endodontic journal, 45(5), 439-448.

Leite, M. L., Soares, D. G., Anovazzi, G., Anselmi, C., Hebling, J., \& de Souza Costa, C. A. (2021). Fibronectin-loaded Collagen/Gelatin Hydrogel Is a Potent Signaling Biomaterial for Dental Pulp Regeneration. Journal of endodontics, 47(7), 1110-1117.

Leites, A. B., Baldissera, E. Z., Silva, A. F., Tarquinio, S., Botero, T., Piva, E., \& Demarco, F. F. (2011). Histologic response and tenascin and fibronectin expression after pulp capping in pig primary teeth with mineral trioxide aggregate or calcium hydroxide. Operative dentistry, 36(4), 448-456. 
Lesot, H., Begue-Kirn, C., Kubler, M. D., Meyer, J. M., Smith, A. J., Cassidy, N., \& Ruch, J. V. (1993). Experimental Induction of Odontoblast Differentiation and Stimulation Durante Processos Preparativos. Cells and Materials. 3(2), 201-217.

Lima, R. V., Esmeraldo, M. R., de Carvalho, M. G., de Oliveira, P. T., de Carvalho, R. A., da Silva, F. L., Jr, \& de Brito Costa, E. M. (2011). Pulp repair after pulpotomy using different pulp capping agents: a comparative histologic analysis. Pediatric dentistry, 33(1), 14-18.

Marques, M. S., Wesselink, P. R., \& Shemesh, H. (2015). Outcome of Direct Pulp Capping with Mineral Trioxide Aggregate: A Prospective Study. Journal of endodontics, 41(7), 1026-1031.

Martins, C. M., Sasaki, H., Hirai, K., Andrada, A. C., \& Gomes-Filho, J. E. (2016). Relationship between hypertension and periapical lesion: an in vitro and in vivo study. Brazilian oral research, 30(1), e78.

Minamikawa, H., Yamada, M., Deyama, Y., Suzuki, K., Kaga, M., Yawaka, Y., \& Ogawa, T. (2011). Effect of N-acetylcysteine on rat dental pulp cells cultured on mineral trioxide aggregate. Journal of endodontics, 37(5), 637-641.

Minic, S., Florimond, M., Sadoine, J., Valot-Salengro, A., Chaussain, C., Renard, E., \& Boukpessi, T. (2021). Evaluation of Pulp Repair after Biodentine ${ }^{\mathrm{TM}}$ Full Pulpotomy in a Rat Molar Model of Pulpitis. Biomedicines, 9(7), 784.

Moradi, S., Saghravanian, N., Moushekhian, S., Fatemi, S., \& Forghani, M. (2015). Immunohistochemical Evaluation of Fibronectin and Tenascin Following Direct Pulp Capping with Mineral Trioxide Aggregate, Platelet-Rich Plasma and Propolis in Dogs' Teeth. Iranian endodontic journal, 10(3), $188-192$.

Murray, G. G., Teixeira, L. M., Oliveira, D. L., Jacomini, L. M., \& Silva, S. R. (2003). Biocompatibility evaluation of Biodentine in subcutaneous tissue of rats. International Endodontic Journal, 36(2), 106-116.

Nowicka, A., Wilk, G., Lipski, M., Kołecki, J., \& Buczkowska-Radlińska, J. (2015). Tomographic Evaluation of Reparative Dentin Formation after Direct Pulp Capping with $\mathrm{Ca}(\mathrm{OH}) 2$, MTA, Biodentine, and Dentin Bonding System in Human Teeth. Journal of endodontics, 41(8), 1234-1240.

Paranjpe, A., Zhang, H., \& Johnson, J. D. (2010). Effects of mineral trioxide aggregate on human dental pulp cells after pulp-capping procedures. Journal of endodontics, 36(6), 1042-1047.

Parirokh, M., Torabinejad, M., \& Dummer, P. (2018). Mineral trioxide aggregate and other bioactive endodontic cements: an updated overview - part I: vital pulp therapy. International endodontic journal, 51(2), 177-205.

Piva, E., Tarquinio, S. B., Demarco, F. F., Silva, A. F., \& de Araujo, V. C. (2006). Immunohistochemical expression of fibronectin and tenascin after direct pulp capping with calcium hydroxide. Oral Surgery, Oral Medicine, Oral Pathology and Oral Radiology, 102(4), 66-71.

Sage, E. H., \& Bornstein, P. (1991). Extracellular proteins that modulate cell-matrix interactions. SPARC, tenascin, and thrombospondin. The Journal of biological chemistry, 266(23), 14831-14834.

Salako, N., Joseph, B., Ritwik, P., Salonen, J., John, P., \& Junaid, T. A. (2003). Comparison of bioactive glass, mineral trioxide aggregate, ferric sulfate, and formocresol as pulpotomy agents in rat molar. Dental traumatology: official publication of International Association for Dental Traumatology, 19(6), 314-320.

Schuurs, A. H. B., Gruythuysen, R. J. M., \& Wesselink, P. R. (2000). Pulp capping with adhesive resinbased composite versus calcium hydroxide: a review. Endodontics \& dental traumatology, 16, 240-250.

Shayegan, A., Jurysta, C., Atash, R., Petein, M., \& Abbeele, A. V. (2012). Biodentine used as a pulp-capping agent in primary pig teeth. Pediatric Dentistry, 34(7), 202-208

Shrestha, P., Sumitomo, S., Lee, C. H., Nagahara, K., Kamegai, A., Yamanaka, T., Takeuchi, H., Kusakabe, M., \& Mori, M. (1996). Tenascin: growth and adhesion modulation--extracellular matrix degrading function: an in vitro study. European journal of cancer. Part B, Oral oncology, 32(2), 106-113.

Silva, L., Lopes, Z., Sá, R. C., Novaes Júnior, A. B., Romualdo, P. C., Lucisano, M. P., Nelson-Filho, P., \& Silva, R. (2019). Comparison of apical periodontitis repair in endodontic treatment with calcium hydroxide-dressing and aPDT. Brazilian oral research, 33, e092.

Tawil, P. Z., Duggan, D. J., \& Galicia, J. C. (2015). Mineral trioxide aggregate (MTA): its history, composition, and clinical applications. Compendium of continuing education in dentistry (Jamesburg, N.J. 1995), 36(4), 247-264.

Thesleff, I., Mackie, E., Vainio, S., \& Chiquet-Ehrismann, R. (1987). Changes in the distribution of tenascin during tooth development. Development (Cambridge, England), 101(2), 289-296.

Torabinejad, M., Parirokh, M., \& Dummer, P. (2018). Mineral trioxide aggregate and other bioactive endodontic cements: an updated overview - part II: other clinical applications and complications. International endodontic journal, 51(3), 284-317.

Tziafas, D. (1994). Mechanisms controlling secondary initiation of dentinogenesis: a review. International Endodontic Journal, 27(2), 61-74.

Tziafas, D., Alvanou, A., Panagiotakopoulos, N., Smith, A. J., Lesot, H., Komnenou, A., \& Ruch, J. V. (1995). Induction of odontoblast-like cell differentiation in dog dental pulps after in vivo implantation of dentine matrix components. Archives of oral biology, 40(10), 883-893.

Valentim, D., Bueno, C. R. E., Marques, V. A. S., Benetti, F., Vasques, A. M. V., Cury, M. T. S., Silva, A. C.R., jacinto, R. C., Sivieri-Araujo, G., Cintra, L. T. A., \& Dezan-Junior, E. (2021). Avaliação da biocompatibilidade de cimentos reparadores biocerâmicos: Estudo in vivo em ratos wistar. Research, Society and Development, 10(7), 1-10.

Yaemkleebbua, K., Osathanon, T., Nowwarote, N., Limjeerajarus, C. N., \& Sukarawan, W. (2019). Analysis of hard tissue regeneration and Wnt signalling in dental pulp tissues after direct pulp capping with different materials. International endodontic journal, 52(11), 1605-1616. 
Research, Society and Development, v. 10, n. 10, e424101018480, 2021

(CC BY 4.0) | ISSN 2525-3409 | DOI: http://dx.doi.org/10.33448/rsd-v10i10.18480

Yoshiba, K., Yoshiba, N., Nakamura, H., Iwaku, M., Ozawa, H. (1996). Immunolocalization of fibronectin during reparative dentinogenesis in human teeth after pulp capping with calcium hydroxide. Journal of Dental Research, 75(8), 1590-1597.

Youssef, A. R., Emara, R., Taher, M. M., Al-Allaf, F. A., Almalki, M., Almasri, M. A., \& Siddiqui, S. S. (2019). Effects of mineral trioxide aggregate, calcium hydroxide, biodentine and Emdogain on osteogenesis, Odontogenesis, angiogenesis and cell viability of dental pulp stem cells. BMC oral health, 19(1), 133.

Zarrabi, M. H., Javidi, M., Jafarian, A. H., \& Joushan, B. (2011). Immunohistochemical expression of fibronectin and tenascin in human tooth pulp capped with mineral trioxide aggregate and a novel endodontic cement. Journal of endodontics, 37(12), 1613-1618. 